\title{
Erratum: Measurement of Preheat Due to Nonlocal Electron Transport in Warm Dense Matter \\ [Phys. Rev. Lett. 120, 025002 (2018)]
}

\author{
K. Falk $\odot$, M. Holec, C. J. Fontes, C. L. Fryer, C. W. Greeff, H. M. Johns, D. S. Montgomery, D. W. Schmidt, and M. Šmíd
}

(Received 1 April 2020; accepted 3 April 2020; published 15 April 2020)

DOI: 10.1103/PhysRevLett.124.159901

Several errors in the Letter have been discovered during post-processing and new analysis after the publication date. Incorrect pressure units were accidentally quoted in Fig. 4. In the published Letter, we discuss the properties of compressed $\mathrm{CH}$ foam after the laser drive was off, thus it should be correctly referred to as a "blast wave." The correct units should be "p [[Mbar/10]." Definitions of all symbols with missing description in the FLYCHK simulations: $T$ is the temperature, $\rho$ is the mass density, and $L$ length of the plasma in the simulation cell. $L$ is not to be confused with the temperature length scale used in the following section describing the PETE simulations. Corrections also include some problems with the analysis of the experimental data and the simulations.

New analysis finds that the original conclusion that the elevated heating in compressed $\mathrm{CH}$ foam was caused by nonlocal electron transport was incorrect. A mistake in our treatment of the energy source [Eq. (11) of [1] ] was uncovered during the investigation of the PETE simulations, where the way we introduced $\lambda^{e}$ lead to $283 \times$ increased energy source, and, consequently, an unrealistically large electron heat flux was generated. Results of nonlocal simulations with a corrected scaling provide a good match to the simulations with heat diffusion. A new kinetic simulation using a Schurtz-NicolaiBusque model [2] was performed, see Fig. 1. Even though we observe a nonlocal transport of electrons originating from the shock, we conclude that they do not introduce any significant effect on the preheat, because the heat flux $5 \mu \mathrm{m}$ ahead of the shock is $10^{-10} \times$ smaller than the heat flux in the shock. Furthermore, upon revisited SOP data analysis the existence of preheat is also inconclusive. The main conclusion of the Letter has changed and thus its title is no longer representative of the work. A more suitable title would be this: observation of abnormally elevated heating in dynamically compressed $\mathrm{CH}$ foam resulting from improved laser drive smoothness.

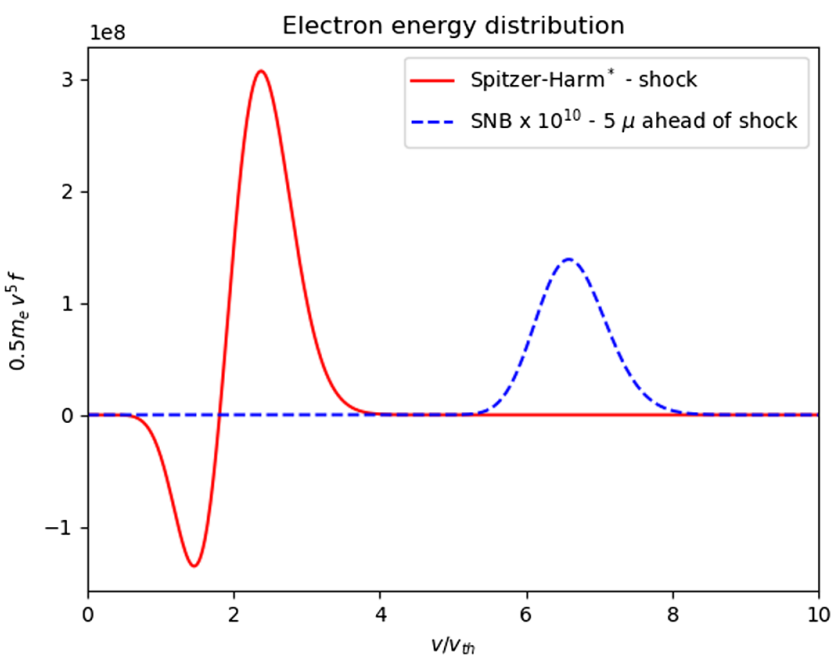

FIG. 1. Energetic distribution of electrons obtained by SNB model inside of the shock (solid red line) and $5 \mu \mathrm{m}$ ahead of the shock. A corresponding heat flux at $5 \mu \mathrm{m}$ ahead of the shock is approximately $10^{-10} \times$ smaller than the heat flux inside of the shock, which perfectly matches the Spitzer-Härm diffusion.

[1] Holec, J. Nikl, and S. Weber, Phys. Plasmas 25, 032704 (2018).

[2] G. Schurtz, P. Nicolai, and M. Busquet, Phys. Plasmas 7, 4238 (2000). 